$0.01 \mathrm{M}$-phosphate buffer, the cyclase was retained and enzymically active on assay in the same buffersalt solution. By contrast, enzyme chromatographed in the absence of $\mathrm{KCl}$ was eluted with the void volume and showed activity only when deoxycholate and $\mathrm{KCl}$ were present in the assay system. Further, it was observed that both $\mathrm{KCl}$ and deoxycholate are required to prevent enzyme activity from sedimenting on high-speed centrifugation. In the absence of salt the enzyme appears to aggregate to an insoluble, catalytically inactive species. Under appropriate conditions the aggregation process is reversible. The molecular weight of the dissociated active form of the enzyme is estimated to be about 90000 , whereas the inactive aggregate ranges in molecular weight from 100000 to 400000 .

A separate enzyme system catalysing the conversion of squalene into 2,3-oxidosqualene was demonstrated as follows. Rat liver microsomes were heated to $50^{\circ}$ for 5 min., combined with the soluble $105000 \mathrm{~g}$ supernatant, and then incubated in air with squalene and NADPH. A compound accumulated, which was identified as 2,3-oxidosqualene. No lanosterol was formed under these conditions. The soluble fraction of the rat liver homogenate contains one or two unidentified factors necessary for squalene epoxide formation. Carbon monoxide or potassium cyanide failed to inhibit epoxidase activity.

Corey, E. J., Russey, W. E. \& Ortiz de Montellano, P. R. (1966). J. Amer. chem. Soc. 88, 4750.

Dean, P. D. G., Ortiz de Montellano, P. R., Bloch, K. \& Corey, E. J. (1967). J. biol. Chem. 242, 3014.

Van Tamelen, E. E. \& Curphey, T. J. (1962). Tetrahedron Lett. p. 121.

Van Tamelen, E. E., Willet, J. D., Clayton, R. B. \& Lord, K. E. (1966). J. Amer. chem. Soc. 88, 4752.

\section{Sterol Biosynthesis}

By L. J. GoAD. (Department of Biochemistry, University of Liverpool)

In the past few years rapid progress has been made in the elucidation of various problems concerned with the final stages of sterol biosynthesis. A number of these investigations have been facilitated by the use of the dual-labelled samples of mevalonic acid developed by J. W. Cornforth and G. Popják. Such studies reveal that, although the biosynthetic route follows a basic pattern, interesting and unexpected divergencies occur in different classes of organism. The most striking example is the nature of the precursor produced by 2,3oxidosqualene cyclization. In animals lanosterol is firmly established as the triterpene that is converted into cholesterol. Similarly, in yeast lanosterol has been shown to act as the precursor of ergosterol. By contrast, lanosterol is of very rare occurrence in higher plants or algae and is apparently only found in some members of the Euphorbiaceae (Ponsinet \& Ourisson, 1968). However, the related triterpene, cycloartenol, is of widespread distribution and becomes rapidly labelled after administration of radioactive mevalonate or acetate to a number of higher plants (Benveniste, Hirth \& Ourisson, 1966; Ehrhardt, Hirth \& Ourisson, 1967; Goad \& Goodwin, 1966) and to the alga Fucus spiralis (Goad \& Goodwin, 1969). This led to the suggestion that in plants cycloartenol is produced rather than lanosterol (Ardenne, Osske, Schreiber, Steinfelder \& Tümmler, 1965 ; Benveniste et al. 1966). Evidence supporting this proposal has now been provided by the conversion of labelled cycloartenol and 24-methylenecycloartanol into phytosterol by the phytoflagellate Ochromonas malhamensis (Hall, Smith, Goad \& Goodwin, 1969) and the accumulation of cycloartenol during anaerobic incubation of 2,3-oxidosqualene with $O$. malhamensis homogenates (H. H. Rees, L. J. Goad \& T. W. Goodwin, unpublished work). However, labelled lanosterol is also converted into phytosterol by $O$. malhamensis and also by Euphorbia peplus (Baisted, Gardner \& McReynolds, 1968), possibly indicating a lack of enzyme specificity in these organisms.

Mevalonate labelled stereospecifically at C-2 has been employed to examine hydrogen elimination during migration of the $\Delta^{8}$ bond to $\Delta^{7}$ in various sterols. Formation of cholesterol by liver and poriferasterol by $O$. malhamensis resulted in elimination of the $7 \beta$ hydrogen atom (Canonica et al. 1968a; Caspi, Greig, Ramm \& Varma, 1968; Smith, Goad \& Goodwin, 1968a; Gibbons, Goad \& Goodwin, 1968a), but with a yeast homogenate the $7 \propto$ hydrogen atom was lost (Caspi \& Ramm, 1969). A further difference between a fungus and an alga has been observed in the formation of the sterol $\Delta^{22}$ bond. In Aspergillus fumugatus the 22 proS and 23 pro $S$ hydrogen atoms were lost during ergosterol production (Bimpson, Goad \& Goodwin, 1969), whereas in $O$. malhamensis the 22 pro $R$ and 23 pro $R$ hydrogen atoms were removed in poriferasterol formation (Smith et al. 1968a,b). Finally, the use of $\left[2 .{ }^{14} \mathrm{C},(2 S)-2-{ }^{3} \mathrm{H}_{1}\right] \mathrm{mevalonate}$ has led to the discovery that the $15 \alpha$ hydrogen atom is eliminated during the conversion of lanosterol into cholesterol (Canonica et al. 1968b; Gibbons, Goad \& Goodwin, $1968 b$ ), probably as a result of C-14 demethylation and with the formation of a sterol $\Delta^{\mathbf{8 , 1 4} \text {-diene }}$ intermediate (Canonica et al. 1968c; Akhtar, Watkinson, Rahimtula, Wilton \& Munday, 1968; Lutsky \& Schroepfer, 1968).

Our investigations have been supported by the Science Research Council and by Unilever Ltd. 
Akhtar, M., Watkinson, I. A., Rahimtula, A. D., Wilton, D. C. \& Munday, K. A. (1968). Chem. Commun. p. 1406. Ardenne, M. von., Osske, G., Schreiber, K., Steinfelder, K. \& Tümmler, R. (1965). Kulturfplanze, 13, 102.

Baisted, D. J., Gardner, R. C. \& McReynolds, C. A. (1968). Phytochemistry, 7, 945.

Benveniste, P., Hirth, L. \& Ourisson, G. (1966). Phytochemistry, 5, 45.

Bimpson, T., Goad, L. J. \& Goodwin, T. W. (1969). Chem. Commun. (in the Press).

Canonica, L., Fiechi, A., Kienle, M. G., Scala, A., Galli, G., Paoletti, E. G. \& Paoletti, R. (1968a). Steroids, 12, 445.

Canonica, L., Fiechi, A., Kienle, M. G., Scala, A., Galli, G., Paoletti, E. G. \& Paoletti, R. (1968b). J. Amer. chem. Soc. 90, 3597.

Canonica, L., Fiechi, A., Kienli, M. G., Scala, A., Galli, G., Paoletti, E. G. \& Paoletti, R. (1968c). J. Amer. chem. Soc. 90, 6532 .

Caspi, E., Greig, J. B., Ramm, P. J. \& Varma, K. R. (1968). Tetrahedron Lett. p. 3829.

Caspi, E. \& Ramm, P. J. (1969). Tetrahedron Lett. p. 181.

Ehrhardt, J. D., Hirth, L. \& Ourisson, G. (1967). Phytochemistry, 6, 815 .

Gibbons, G. F., Goad, L. J. \& Goodwin, T. W. (1968a). Chem. Commun. p. 1212.

Gibbons, G. F., Goad, L. J. \& Goodwin, T. W. (1968b). Chem. Commun. p. 1458.

Goad, L. J. \& Goodwin, T. W. (1966). Biochem. J. 99, 735.

Goad, L. J. \& Goodwin, T. W. (1969). Europ. J. Biochem. (in the Press).

Hall, J., Smith, A. R. H., Goad, L. J. \& Goodwin, T. W. (1969). Biochem. J. 112, 129.

Lutsky, B. N. \& Schroepfer, G. J. (1968). Biochem. biophys. Res. Commun. 33, 492.

Ponsinet, G. \& Ourisson, G. (1968). Phytochemistry, 7, 89.

Smith, A. R. H., Goad, L. J. \& Goodwin, T. W. (1968a). Chem. Commun. p. 1259.

Smith, A. R. H., Goad, L. J. \& Goodwin, T. W. (1968b). Chem. Commun. p. 926.

\section{Nature and Distribution of Terpene Quinones}

By O. Wrss and U. GLoor. (Department of Vitamin, and Nutritional Research, $F$. Hoffmann-La Roche \& Co. Ltd., Basle, Switzerland)

The terpene quinones observed in Nature are, according to their quinone component, divided into phylloquinone, menaquinones, ubiquinones and plastoquinones. Variations within the particular groups are caused by the length of the side chain, the number of double bonds and, for a few of them, the oxygen function within the side chain.

Plants for phylloquinone and micro-organisms for menaquinones were identified exclusively as places of origin. However, animals, micro-organisms and plants are able to synthesize ubiquinones.

Animals also ingest terpene quinones with their food. Thus the question arises how the administered quinones are distributed, metabolized and excreted. Two methods are particularly suitable for observing the distribution in the various organs and tissues:
(1) administration of labelled compounds and localization of the radioactivity in the tissues after combustion; (2) radioautographic studies in the whole animal.

Results obtained up to now in the rat show that distribution and excretion in certain organs depend mainly on the length of the side chain. The degree of saturation of the side chain, too, seems to exert a certain influence.

Excretion takes place via bile and urine. The radioactive quinones are apparently excreted unchanged in the bile; but metabolites are found in the urine. Independent of the nature of the quinone component and the length and degree of saturation of the side chains, the latter are shortened to seven carbon atoms, and $\gamma$-lactones are formed.

Ubiquinones and plastoquinones were also studied with respect to their intracellular distribution. In plants as well as in animal cells, mitochondria are enriched with ubiquinone; however, plastoquinones are found in the chloroplasts.

\section{The Biosynthesis of Terpenoid Quinones $\dagger$}

By HARRY RUDNEy. (Department of Biological Chemistry, College of Medicine, University of Cincinnati, Cincinnati, Ohio, U.S.A.)

Most of the work on this subject before 1966 has been well documented in monographs and reports of the proceedings of several symposia (see, e.g., Morton, 1965; Pridham, 1967; Harris, 1966). This presentation will deal mainly with the synthesis of ubiquinone and reference will be made to other quinones where significant differences appear.

The methyl groups of ubiquinone, both ring methyl and $O$-methyl, have been shown to arise from $S$-adenosylmethionine and the polyprenyl side chain from derivatives of mevalonate. The latter can exist in a variety of chain lengths with one to twelve isoprenoid unit groups in the side chain, depending on the source from which it is derived. The benzoquinone ring, however, has been shown to arise via $\mathrm{POB},{ }^{*}$ and it is presumed that the latter is generated via the shikimate and chorismate pathways. The use of POB has provided a unique probe for tracing the metabolic sequences in ubiquinone biosynthesis, and has played the same role that mevalonate has in steroid and polyisoprenoid synthesis. The use of $p$-hydroxybenzaldehyde and $\mathrm{POB}$ as precursors of ubiquinone in Rhodospirillum rubrum (Parson \& Rudney, 1965a,b)

* Abbreviations: POB, $p$-hydroxybenzoate; IPP, isopentenyl pyrophosphate.

†Recent work described from the author's laboratory was supported by grants from the National Institutes of Health NIAM-AM-12463 and the Life Insurance Medical Research Fund. 